\title{
Closed-form solution of a thermocapillary free-film problem due to Pukhnachev
}

\author{
BRIAN R. DUFFY, MATTHIAS LANGER and STEPHEN K. WILSON ${ }^{1}$ \\ Department of Mathematics and Statistics, University of Strathclyde, \\ 26 Richmond Street, Glasgow G1 1XH, United Kingdom
}

Email: b.r.duffy@strath.ac.uk, m.langer@strath.ac.uk, s.k.wilson@strath.ac.uk

(Received 4th July 2014; revised 18th December 2014 and 18th February 2015)

\begin{abstract}
We consider the steady two-dimensional thin-film version of a problem concerning a weightless non-isothermal free fluid film subject to thermocapillarity, proposed and analysed by Pukhnachev and co-workers. Specifically, we extend and correct the paper by Karabut and Pukhnachev (J. App. Mech. Tech. Phys. 49, 568-579, 2008), in which the problem is solved numerically, and in which it is claimed that there exists a unique solution for any value of a prescribed heat-flux parameter in the model. We present a closed-form (parametric) solution of the problem, and from this show that, on the contrary, solutions exist only when the heat-flux parameter is less than a critical value found numerically by Karabut and Pukhnachev, and that when this condition is satisfied there are in fact two solutions, one of which recovers that obtained numerically by Karabut and Pukhnachev, the other being new.
\end{abstract}

Key words: free film; non-isothermal flow; thermocapillarity, Pukhnachev

\section{Problem statement}

In a series of papers Pukhnachev ${ }^{2}$ and co-workers (Pukhnachov [13], Pukhnachev \& Dubinkina [15], Pukhnachev [14] and Karabut \& Pukhnachev [8]), motivated by the behaviour of free films of fluid in foams, proposed and analysed a model of a weightless non-isothermal film of incompressible viscous fluid that spans a hollow cylinder on which a heat-flux distribution is prescribed, the upper and lower surfaces of the film being free. The film is subject to thermocapillarity, the surface tension of the fluid being taken to vary linearly with temperature (but with its density, viscosity and thermal conductivity taken to be constants). A flow is therefore generated within the film, the energy of the flow being supplied by the prescribed heat input/output at the cylindrical boundary.

In particular, Pukhnachev and co-workers considered the situation in which the film is thin, with thickness much less than a typical diameter of the cylinder, and in which the appropriate reduced Reynolds and Péclet numbers are small, so that the lubrication approximation may be used. The cylinder, which may in general be of arbitrary crosssection, is taken to have generators in the $z$ direction referred to Cartesian coordinates Oxyz, and the film is taken to be symmetric about the plane $z=0$, with its upper and lower free surfaces at $z= \pm h(x, y, t)$, where $t$ denotes time. Interestingly, as Pukhnachev [14] described, within the framework of the thin-film model (and very differently from flows of

\footnotetext{
${ }^{1}$ Author for correspondence.

${ }^{2}$ This author's name has been transliterated into English as both Pukhnachev and Pukhnachov.
} 
thin films in contact with solid substrates), the shape of the free surfaces of the film may be determined without detailed knowledge of the dependence of the fluid velocity on $z$.

Pukhnachev [14] showed that in the case when the upper and lower free surfaces are thermally insulated (his "Problem B") and the flow is steady the non-dimensional problem for the thickness $2 h$ and depth-averaged temperature $T(x, y)$ of the film reduces to the system

$$
\nabla \cdot\left(h \nabla \nabla^{2} h\right)=\gamma \nabla^{2} T, \quad \nabla \cdot(h \nabla T)=0 \quad \text { in } \quad \Omega,
$$

to be solved subject to the boundary conditions

$$
\frac{\partial h}{\partial n}=0, \quad h \frac{\partial \nabla^{2} h}{\partial n}=\gamma \frac{\partial T}{\partial n}, \quad h \frac{\partial T}{\partial n}=g \quad \text { on } \quad \partial \Omega,
$$

the prescribed-volume condition

$$
\iint_{\Omega} h \mathrm{~d} S=V
$$

and a normalisation condition

$$
\iint_{\Omega} T \mathrm{~d} S=0
$$

where $\Omega$ denotes the interior of the cross-section of the cylindrical boundary in the plane $z=0, \partial \Omega$ denotes the plane curve that bounds $\Omega, \nabla$ denotes the two-dimensional gradient in $\Omega, \partial / \partial n$ denotes differentiation in the direction of the normal to $\partial \Omega$ outward from the cylinder, $V$ is the volume of fluid in the film, $\gamma(>0)$ is an effective Marangoni number, and $g$ is a prescribed function which is subject to the compatibility condition

$$
\int_{\partial \Omega} g \mathrm{~d} \ell=0
$$

$\ell$ denoting arc length along $\partial \Omega$. In $(1.2)_{1}$ the contact angle of the fluid where the free surfaces meet the cylindrical boundary has been taken to be $\pi / 2$.

As Pukhnachev [14] described, in the case when the cylindrical boundary comprises the planes $x=0$ and $x=1$ (so that $\Omega$ is the infinite strip $0 \leq x \leq 1, z=0$, and $\partial \Omega$ reduces to the lines $x=0, z=0$ and $x=1, z=0$ ), and the function $g$ in (1.2) is a constant heat-flux parameter $-q$ on $x=0$ and $+q$ on $x=1$, the problem becomes two-dimensional, with both $h$ and $T$ independent of $y$, and with the fluid film occupying $-h(x) \leq z \leq h(x)$ for $0 \leq x \leq 1$. Figure 1 shows a sketch of the geometry in the $x, z$ plane in this case; it is this two-dimensional version of the problem that we consider in the present study. In that case one integration of each of the equations in (1.1) subject to (1.2) leads to

$$
h h^{\prime \prime \prime}=\gamma T^{\prime}, \quad h T^{\prime}=q, \quad x \in(0,1),
$$

where a prime denotes differentiation with respect to argument. Thus the free-surface profiles $z= \pm h(x)$ satisfy

$$
\begin{array}{cc}
h^{2} h^{\prime \prime \prime} & =-b, \quad x \in(0,1) \\
h^{\prime}(0) & =0, \quad h^{\prime}(1)=0,
\end{array}
$$

and

$$
\int_{0}^{1} h(x) \mathrm{d} x=1
$$




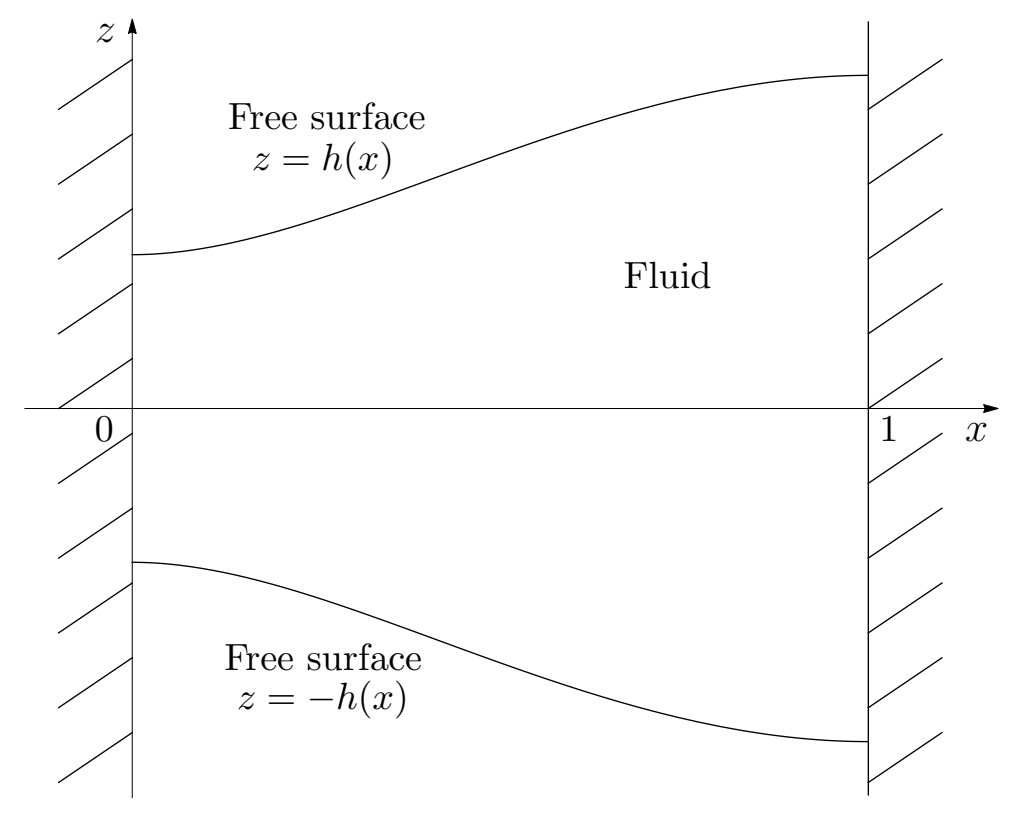

Figure 1: Sketch of the steady two-dimensional version of a problem proposed and analysed by Pukhnachev and co-workers: a non-isothermal thin fluid film in $0 \leq x \leq 1,-h(x) \leq z \leq$ $h(x)$, whose free surfaces $z= \pm h(x)$ are subject to thermocapillarity.

where the constant heat-flux parameter $b$, which is proportional to $q$, may be taken to be non-negative without loss of generality (see [14]). Equation (1.9) comes from (1.3), with $V$ now referring to volume per unit width in the $y$ direction, and taken to be unity without loss of generality.

With $h(x)$ determined from (1.7)-(1.9) the temperature $T(x)$ is given by

$$
T(x)=-|q| \int_{0}^{x} \frac{\mathrm{d} \tilde{x}}{h(\tilde{x})}+|q| \int_{0}^{1} \int_{0}^{\hat{x}} \frac{\mathrm{d} \tilde{x}}{h(\tilde{x})} \mathrm{d} \hat{x}
$$

satisfying the normalisation condition

$$
\int_{0}^{1} T(x) \mathrm{d} x=0 .
$$

In the present paper we provide a closed-form (parametric) solution of the two-dimensional problem defined by (1.7)-(1.11), and describe its properties. Specifically, we extend and correct the paper by Karabut \& Pukhnachev [8] in which the problem is solved numerically, and in which it is claimed that there exists a unique solution for any value of $b$. We present our solution in Section 2, and from this we show that, on the contrary, solutions exist only when $b$ does not exceed a critical value $b_{c}$ found numerically by Karabut \& Pukhnachev [8], and that when $b \leq b_{\mathrm{c}}$ there are in fact two solutions, one of which recovers that obtained numerically by Karabut \& Pukhnachev [8], the other being new. In Section 3 some properties of the solutions are discussed, in Section 4 comparison is made with the analysis of Karabut \& Pukhnachev [8], and in Section 5 the limit $b \rightarrow 0$ is considered. In 
an appendix we derive asymptotic expansions of certain integrals that are used to obtain our main results.

It is worth noting that the ordinary differential equation in (1.7) arises in many other contexts involving thin films, and so has been studied extensively; see, for example, the papers by Voinov [18], Tuck \& Schwartz [17], Duffy \& Wilson [4], Eggers [5, 6], Limat \& Stone [10], Bonn et al. [2], Neogi [11], Chan, Gueudré \& Snoeijer [3], Karpitschka \& Riegler [9], Janeček et al. [7] and Snoeijer \& Andreotti [16]. In particular, Duffy \& Wilson [4] discussed the general solution of this differential equation in some detail.

\section{Solution of the two-dimensional problem (1.7)-(1.11)}

When $b=0$ the (unique) solution of (1.7)-(1.11) is simply

$$
h \equiv 1, \quad T=|q|\left(\frac{1}{2}-x\right),
$$

and so from now on we take $b>0$, in general.

\section{$2.1 \quad$ General solution}

As discussed by, for example, Duffy \& Wilson [4], the substitution

$$
\frac{\mathrm{d} x}{\mathrm{~d} s}=\left(\frac{2}{b}\right)^{1 / 3} \frac{1}{z(s)^{2}}, \quad h=\frac{1}{z(s)^{2}}
$$

in terms of a parameter $s$, reduces the differential equation (1.7) to

$$
\frac{\mathrm{d}}{\mathrm{d} s}\left(\frac{1}{z} \frac{\mathrm{d}^{2} z}{\mathrm{~d} s^{2}}\right)=1
$$

whose solution may, without loss of generality, be written as

$$
z(s)=\alpha \operatorname{Ai}(s)+\beta \operatorname{Bi}(s),
$$

where Ai and Bi denote the usual Airy functions (Abramowitz and Stegun [1] or the NIST Handbook of Mathematical Functions [12]), and $\alpha$ and $\beta$ are arbitrary constants (the third integration constant having been set to zero since it leads only to a shift in the parameter $s$ ). From (2.2) the general solution of (1.7) may be written in the parametric form

$$
x=\left(\frac{2}{b}\right)^{1 / 3} \int_{s_{0}}^{s} \frac{1}{z(\tilde{s})^{2}} \mathrm{~d} \tilde{s}, \quad h=\frac{1}{z(s)^{2}},
$$

where the constant $s_{0}$ denotes the value of $s$ when $x=0$, so that $x\left(s_{0}\right)=0$. Utilising the relations

$$
\operatorname{Ai}(s) \operatorname{Bi}^{\prime}(s)-\operatorname{Ai}^{\prime}(s) \operatorname{Bi}(s)=\frac{1}{\pi}, \quad \frac{\mathrm{d}}{\mathrm{d} s}\left(\frac{a_{1} \operatorname{Ai}(s)+b_{1} \operatorname{Bi}(s)}{a \operatorname{Ai}(s)+b \operatorname{Bi}(s)}\right)=\frac{a b_{1}-b a_{1}}{\pi[a \operatorname{Ai}(s)+b \operatorname{Bi}(s)]^{2}}
$$


for any constants $a, b, a_{1}$ and $b_{1}$, we may perform the quadrature in (2.5) to obtain

$$
\begin{aligned}
& x=\left(\frac{2}{b}\right)^{1 / 3} \frac{\pi}{\alpha \beta_{1}-\beta \alpha_{1}}\left(\frac{\alpha_{1} \operatorname{Ai}(s)+\beta_{1} \operatorname{Bi}(s)}{z(s)}-\frac{\alpha_{1} \operatorname{Ai}\left(s_{0}\right)+\beta_{1} \operatorname{Bi}\left(s_{0}\right)}{z\left(s_{0}\right)}\right), \\
& h=\frac{1}{z(s)^{2}}
\end{aligned}
$$

where the constants $\alpha_{1}$ and $\beta_{1}$ are arbitrary except that $\alpha \beta_{1}-\beta \alpha_{1} \neq 0$ (the expression for $x$ in (2.7) being independent of the choice of $\alpha_{1}, \beta_{1}$ ). It will turn out in the present problem that $\alpha \beta \neq 0$, and so without loss of generality we may take $\alpha_{1}=0$ and $\beta_{1} \neq 0$; thus finally we obtain the general solution of (1.7) in the closed (parametric) form

$$
x=\pi\left(\frac{2}{b}\right)^{1 / 3} \frac{\operatorname{Ai}\left(s_{0}\right) \operatorname{Bi}(s)-\operatorname{Bi}\left(s_{0}\right) \operatorname{Ai}(s)}{z\left(s_{0}\right) z(s)}, \quad h=\frac{1}{z(s)^{2}},
$$

where $z$ is given in (2.4). To determine the temperature $T$ in terms of the parameter $s$ we integrate the second equation in (1.6) using (2.2) and then impose the normalisation condition (1.11) to obtain

$$
T=T_{0}-\left(\frac{2}{b}\right)^{1 / 3}|q| s, \quad T_{0}=\left(\frac{2}{b}\right)^{2 / 3}|q| \int_{s_{0}}^{s_{1}} \frac{s}{z(s)^{2}} \mathrm{~d} s=\text { constant }
$$

showing that $T$ is, in fact, simply linear in $s$ (and hence that $T$ could have been used as the independent variable, in place of the parameter $s$ ).

To complete the solution, we must now determine the constants $\alpha, \beta, s_{0}$ and $s_{1}$ such that (1.8) and (1.9) are satisfied. For later use we note that

$$
\frac{\mathrm{d} h}{\mathrm{~d} x}=-2\left(\frac{b}{2}\right)^{1 / 3} \frac{1}{z} \frac{\mathrm{d} z}{\mathrm{~d} s}, \quad \frac{\mathrm{d}^{2} h}{\mathrm{~d} x^{2}}=2\left(\frac{b}{2}\right)^{2 / 3}\left[\left(\frac{\mathrm{d} z}{\mathrm{~d} s}\right)^{2}-s z^{2}\right] .
$$

\subsection{Conditions on $z(s)$}

Denoting by $s_{1}$ the value of $s$ when $x=1$ we have $x\left(s_{1}\right)=1$, which with $(2.5)$ gives

$$
\int_{s_{0}}^{s_{1}} \frac{1}{z(s)^{2}} \mathrm{~d} s=\left(\frac{b}{2}\right)^{1 / 3}
$$

or equivalently with (2.8) gives

$$
\frac{\operatorname{Ai}\left(s_{0}\right) \operatorname{Bi}\left(s_{1}\right)-\operatorname{Bi}\left(s_{0}\right) \operatorname{Ai}\left(s_{1}\right)}{z\left(s_{0}\right) z\left(s_{1}\right)}=\frac{1}{\pi}\left(\frac{b}{2}\right)^{1 / 3} .
$$

From (1.8) and (2.10) we have

$$
\frac{\mathrm{d} z}{\mathrm{~d} s}=0 \quad \text { at } \quad s=s_{0}, \quad s=s_{1},
$$

that is,

$$
\alpha \operatorname{Ai}^{\prime}\left(s_{0}\right)+\beta \operatorname{Bi}^{\prime}\left(s_{0}\right)=0, \quad \alpha \operatorname{Ai}^{\prime}\left(s_{1}\right)+\beta \operatorname{Bi}^{\prime}\left(s_{1}\right)=0 .
$$


Lastly, the prescribed-volume condition (1.9) gives

$$
\int_{s_{0}}^{s_{1}} \frac{1}{z(s)^{4}} \mathrm{~d} s=\left(\frac{b}{2}\right)^{1 / 3} .
$$

With $b(>0)$ prescribed, the parameters $\alpha, \beta, s_{0}$ and $s_{1}$ are to be determined from the algebraic equations (2.11), (2.14) and (2.15), and then the complete solution for $h$ in (2.8) and $T$ in $(2.9)$ is known.

The parameters $\alpha$ and $\beta$ may be eliminated from (2.11), (2.14) and (2.15) to give a pair of simultaneous algebraic equations for $s_{0}$ and $s_{1}$ :

$$
\operatorname{Ai}^{\prime}\left(s_{0}\right) \operatorname{Bi}^{\prime}\left(s_{1}\right)=\operatorname{Bi}^{\prime}\left(s_{0}\right) \operatorname{Ai}^{\prime}\left(s_{1}\right), \quad J_{2}^{2}=\left(\frac{b}{2}\right)^{1 / 3} J_{4},
$$

where we have defined $J_{n}=J_{n}\left(s_{0}, s_{1}\right)$ for $n=2$ and $n=4$ by

$$
J_{n}\left(s_{0}, s_{1}\right):=\int_{s_{0}}^{s_{1}} \frac{\mathrm{d} s}{\left[\operatorname{Bi}^{\prime}\left(s_{1}\right) \operatorname{Ai}(s)-\operatorname{Ai}^{\prime}\left(s_{1}\right) \operatorname{Bi}(s)\right]^{n}} .
$$

Then, with $s_{0}$ and $s_{1}$ known, $\alpha$ and $\beta$ are given by

$$
\begin{aligned}
& \alpha=\left(\frac{2}{b}\right)^{1 / 6} J_{2}^{1 / 2} \operatorname{Bi}^{\prime}\left(s_{1}\right)=\frac{J_{4}^{1 / 2}}{J_{2}^{1 / 2}} \operatorname{Bi}^{\prime}\left(s_{1}\right), \\
& \beta=-\left(\frac{2}{b}\right)^{1 / 6} J_{2}^{1 / 2} \mathrm{Ai}^{\prime}\left(s_{1}\right)=-\frac{J_{4}^{1 / 2}}{J_{2}^{1 / 2}} \mathrm{Ai}^{\prime}\left(s_{1}\right) .
\end{aligned}
$$

We note that in the case $n=2$ the quadrature in (2.17) may be performed explicitly:

$$
J_{2}\left(s_{0}, s_{1}\right)=\pi^{2} \frac{\operatorname{Ai}\left(s_{0}\right) \operatorname{Bi}\left(s_{1}\right)-\operatorname{Bi}\left(s_{0}\right) \operatorname{Ai}\left(s_{1}\right)}{\operatorname{Ai}\left(s_{0}\right) \operatorname{Bi}^{\prime}\left(s_{1}\right)-\operatorname{Bi}\left(s_{0}\right) \operatorname{Ai}^{\prime}\left(s_{1}\right)},
$$

obtained via (2.6) with $a=\operatorname{Bi}^{\prime}\left(s_{1}\right), b=-\mathrm{Ai}^{\prime}\left(s_{1}\right), a_{1}=\operatorname{Bi}\left(s_{1}\right)$ and $b_{1}=\operatorname{Bi}\left(s_{1}\right)$; however, in the case $n=4$ there seems to be no corresponding simple expression for $J_{4}\left(s_{0}, s_{1}\right)$.

\section{$2.3 \quad$ Existence of solutions}

The film thickness $2 h$ must be finite for $0 \leq x \leq 1$, and so $z(s)$ must not vanish for $s_{0} \leq s \leq s_{1}$, that is, $z(s)$ must remain of one sign (which without loss of generality we may take to be positive) between its stationary points at $s=s_{0}$ and $s=s_{1}$. If $\alpha=0$ (so that $z(s) \propto \operatorname{Bi}(s)$ ) or $\beta=0$ (so that $z(s) \propto \operatorname{Ai}(s)$ ) then $z(s)$ has no pairs of stationary points between which it is of one sign; therefore, as indicated earlier, we will always have $\alpha \beta \neq 0$. Since $z(s)$ has stationary points at $s=s_{0}$ and $s=s_{1}$, it must have at least one inflection point in $\left(s_{0}, s_{1}\right)$. Moreover, since $z(s)$ satisfies $z^{\prime \prime}(s)=s z(s)$, and since we require $z>0$, an inflection point can occur only at $s=0$. We deduce that $z(s)$ has a single inflection point at $s=0$, and hence that $s_{0}<0<s_{1}$. It therefore also follows that $z$ has no stationary point in $\left(s_{0}, s_{1}\right)$.

Since $\alpha$ and $\beta$ are non-zero, by (2.14) we have $\operatorname{Bi}^{\prime}\left(s_{0}\right), \operatorname{Bi}^{\prime}\left(s_{1}\right) \neq 0$. Therefore the first equation in $(2.16)$ is equivalent to

$$
\frac{\operatorname{Ai}^{\prime}\left(s_{0}\right)}{\operatorname{Bi}^{\prime}\left(s_{0}\right)}=\frac{\operatorname{Ai}^{\prime}\left(s_{1}\right)}{\operatorname{Bi}^{\prime}\left(s_{1}\right)}
$$




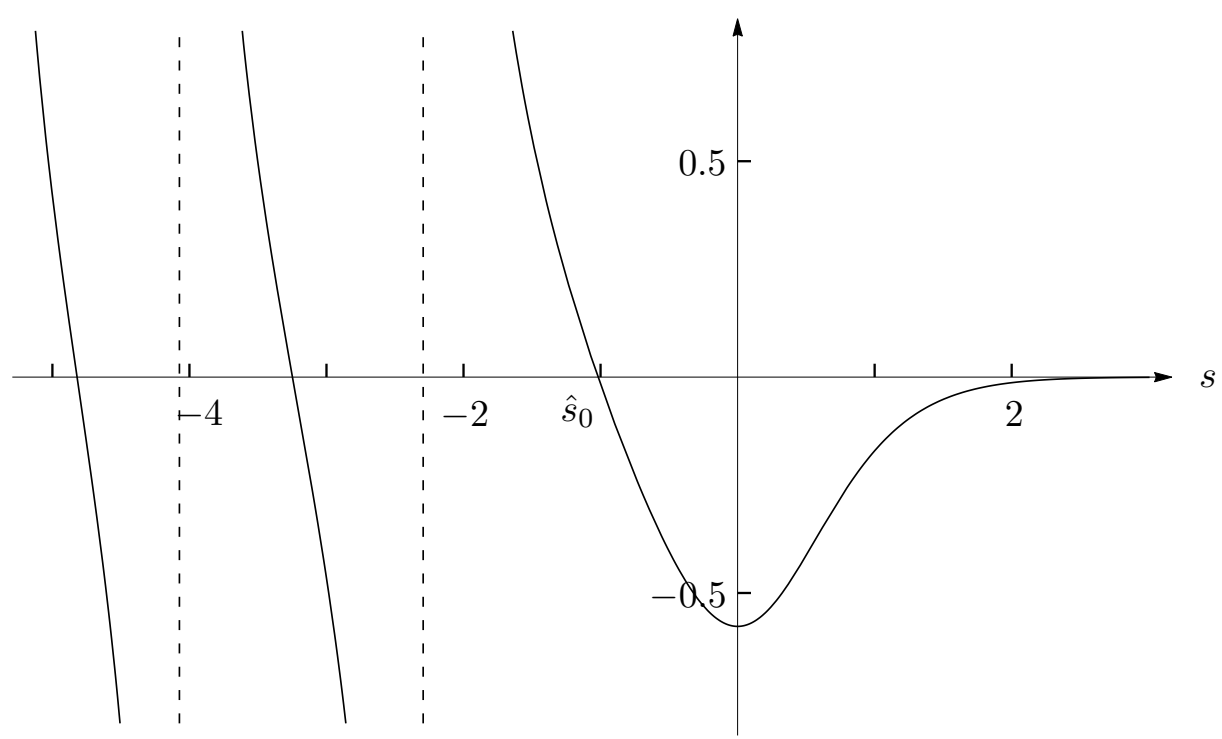

Figure 2: Plot of $\mathrm{Ai}^{\prime}(s) / \mathrm{Bi}^{\prime}(s)$ as a function of $s$. The largest (negative) zero of this function is at $s=\hat{s}_{0} \simeq-1.018793$.

Figure 2 shows a plot of $\operatorname{Ai}^{\prime}(s) / \mathrm{Bi}^{\prime}(s)$ as a function of $s$; the relation

$$
\frac{\mathrm{d}}{\mathrm{d} s}\left(\frac{\operatorname{Ai}^{\prime}(s)}{\operatorname{Bi}^{\prime}(s)}\right)=\frac{s}{\pi\left[\operatorname{Bi}^{\prime}(s)\right]^{2}}
$$

implies that $\operatorname{Ai}^{\prime}(s) / \operatorname{Bi}^{\prime}(s)$ is increasing on the interval $[0, \infty)$ and decreasing on each subinterval of $(-\infty, 0]$ that lies between two neighbouring poles. Let $s=\hat{s}_{0} \simeq-1.018793$ denote the largest (negative) zero of $\operatorname{Ai}^{\prime}(s)$. If $s_{1}$ is given, then $s_{0}$ must be the largest negative solution of $(2.21)$, i.e. $s_{0}$ must be the unique solution of $(2.21)$ in the interval $\left(\hat{s}_{0}, 0\right)$, for if the solution $s_{0}$ were on any "lower" branch (i.e. $s_{0}<\hat{s}_{0}$ ), then the function $z$ would have one or more stationary points in $\left(s_{0}, s_{1}\right)$ (corresponding to solutions of $(2.21)$ on "higher" branches), contradicting our earlier conclusion that $z$ has no such stationary point. Hence we have shown the following lemma.

Lemma 2.1. For given $b>0$ the problem (1.7)-(1.9) has a solution if and only if there exist $s_{1}>0$ and $s_{0} \in\left(\hat{s}_{0}, 0\right)$ such that

$$
\frac{\operatorname{Ai}^{\prime}\left(s_{0}\right)}{\operatorname{Bi}^{\prime}\left(s_{0}\right)}=\frac{\operatorname{Ai}^{\prime}\left(s_{1}\right)}{\operatorname{Bi}^{\prime}\left(s_{1}\right)}, \quad\left(\frac{b}{2}\right)^{1 / 3}=\frac{\left[J_{2}\left(s_{0}, s_{1}\right)\right]^{2}}{J_{4}\left(s_{0}, s_{1}\right)},
$$

where the $J_{n}\left(s_{0}, s_{1}\right)$ are defined in (2.17). In that case a solution is given in closed (parametric) form by

$$
x=\pi\left(\frac{2}{b}\right)^{1 / 3} \frac{\operatorname{Ai}\left(s_{0}\right) \operatorname{Bi}(s)-\operatorname{Bi}\left(s_{0}\right) \operatorname{Ai}(s)}{z\left(s_{0}\right) z(s)}, \quad h=\frac{1}{z(s)^{2}},
$$

where

$$
z(s)=\frac{\left[J_{4}\left(s_{0}, s_{1}\right)\right]^{1 / 2}}{\left[J_{2}\left(s_{0}, s_{1}\right)\right]^{1 / 2}}\left[\operatorname{Bi}^{\prime}\left(s_{1}\right) \operatorname{Ai}(s)-\operatorname{Ai}^{\prime}\left(s_{1}\right) \operatorname{Bi}(s)\right] .
$$

Moreover, the temperature $T$ is given by (2.9). 


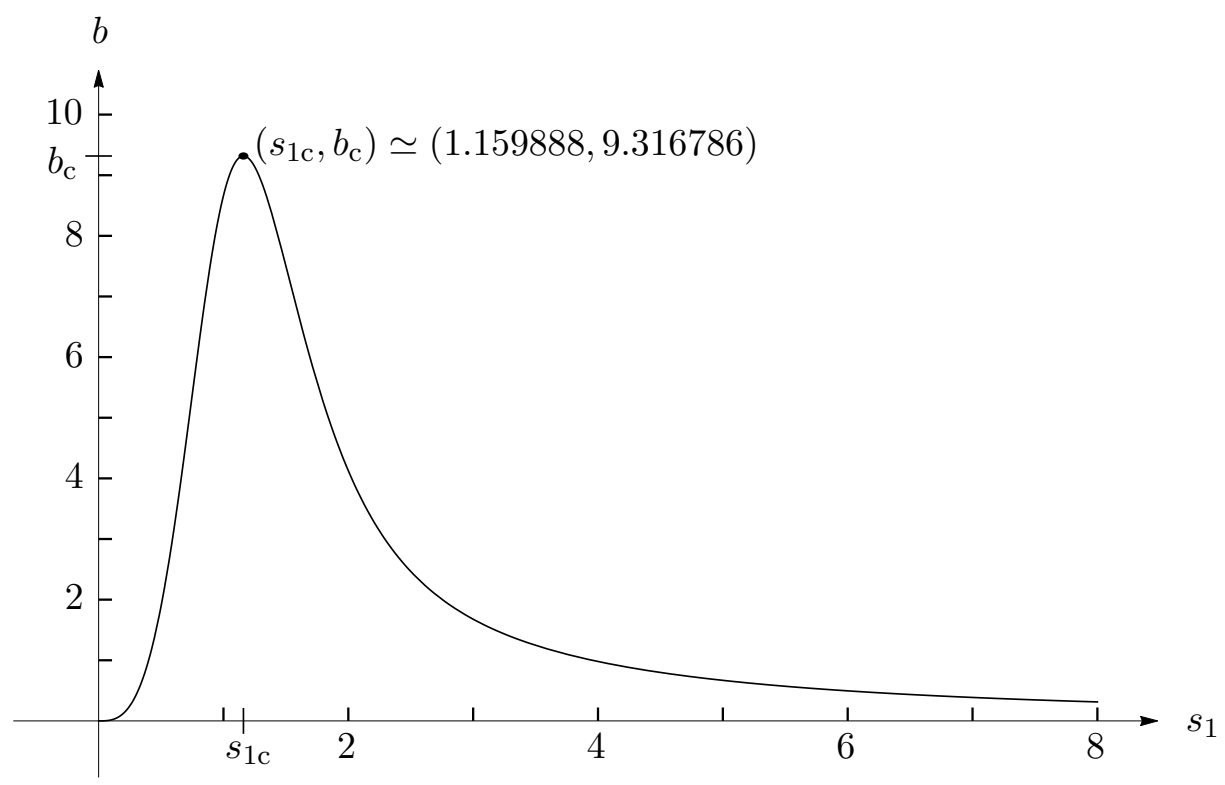

Figure 3: Plot of $b$ as a function of $s_{1}$, indicating, in particular, that there exists a solution only when $0 \leq b \leq b_{\mathrm{c}}$.

Rather than prescribing $b$ and then determining $s_{0}, s_{1}$ and $z$ from (2.23) and (2.25), it is more convenient and numerically more efficient to prescribe $s_{1} \in(0, \infty)$ and then determine $s_{0}$ from $(2.23)_{1}, b$ from $(2.23)_{2}$ and $z$ from $(2.25)$.

Figure 3 shows a plot of $b$ as a function of $s_{1}(>0)$, indicating, in particular, that there exists a critical value $b=b_{\mathrm{c}} \simeq 9.316786$ (see subsection 2.4 below), corresponding to $s_{1}=s_{1 \mathrm{c}} \simeq 1.159888$, such that for $b<b_{\mathrm{c}}$ there are two values of $s_{1}$ that lead to this $b$, i.e. there are two solutions $h$, for $b=b_{\mathrm{c}}$ there is one value of $s_{1}$ and hence one solution for $h$, and for $b>b_{c}$ there is no solution. We now prove rigorously that there is only a finite range of values for $b$ for which there exists a solution.

Theorem 2.2. There exists a critical value $b_{\mathrm{c}}>0$ such that for $b<b_{\mathrm{c}}$ the problem (1.7)(1.9) has at least two solutions and for $b>b_{\mathrm{c}}$ it has no solution.

Proof. First note that the function $b$ depends continuously on $s_{1}$. We consider the asymptotic behaviour of $b$ as $s_{1} \rightarrow 0^{+}$and $s_{1} \rightarrow \infty$.

When $s_{1} \rightarrow 0^{+}$we have straightforwardly

$$
s_{0} \sim-s_{1} \rightarrow 0^{-}, \quad J_{n} \sim 2 \pi^{n} s_{1}, \quad b \sim 16 s_{1}^{3} \rightarrow 0^{+} .
$$

For the behaviour as $s_{1} \rightarrow \infty$ we use Lemma A.1, which implies that

$$
b=2 \frac{J_{2}^{6}}{J_{4}^{3}} \sim 2 \frac{\left(\pi^{2} s_{1}^{-1 / 2}\right)^{6}}{\left(\frac{2}{3} \pi^{4} s_{1}^{-1 / 2}\right)^{3}}=\frac{27}{4} s_{1}^{-3 / 2} \rightarrow 0^{+}, \quad s_{1} \rightarrow \infty .
$$

Hence there exists a value $b_{\mathrm{c}}$ such that for $b>b_{\mathrm{c}}$ there exists no solution for $s_{1}$ (and hence no solution of (1.7)-(1.9)) and for $b<b_{\mathrm{c}}$ there exist at least two different solutions for $s_{1}$. It remains to show that different values of $s_{1}$ with the same $b$ lead to different solutions for 
$h$ and $T$. Assume that this is not the case. Then, for $b<b_{\mathrm{c}}$, there exist $\tilde{s}_{1}>s_{1}$ and $\tilde{s}_{0}<s_{0}$ both of which solve (2.23) and lead to the same functions $h(x)$ and $T(x)$. By (2.9) we then have

$$
T(1)-T(0)=\left(\frac{2}{b}\right)^{1 / 3}|q|\left(s_{0}-s_{1}\right)=\left(\frac{2}{b}\right)^{1 / 3}|q|\left(\tilde{s}_{0}-\tilde{s}_{1}\right),
$$

which is a contradiction.

Figure 3 indicates that there is a fold bifurcation at the critical value $b_{\mathrm{c}}$. In the following we call the set of solutions corresponding to $s_{1}<s_{1 \mathrm{c}}$ the "first family" of solutions and those corresponding to $s_{1}>s_{1 \mathrm{c}}$ the "second family".

\subsection{Critical values of the parameters}

The critical value $b_{\mathrm{c}}$ was obtained numerically by solving (2.23) simultaneously with the condition that $b$, regarded as a function of $s_{1}$, has a maximum $b=b_{\mathrm{c}}$ at some $s_{1}=s_{1 \mathrm{c}}$ (with corresponding $s_{0}=s_{0 \mathrm{c}}$ ), namely

$$
2 J_{4} \frac{\mathrm{d} J_{2}}{\mathrm{~d} s_{1}}=J_{2} \frac{\mathrm{d} J_{4}}{\mathrm{~d} s_{1}}
$$

obtained by differentiation of $(2.23)_{2}$. The derivatives here may be written as

$$
\begin{aligned}
\frac{\mathrm{d} J_{n}}{\mathrm{~d} s_{1}}= & \pi^{n}+\frac{s_{1}\left[\operatorname{Ai}^{\prime}\left(s_{0}\right) \operatorname{Bi}\left(s_{1}\right)-\operatorname{Bi}^{\prime}\left(s_{0}\right) \operatorname{Ai}\left(s_{1}\right)\right]}{s_{0}\left[\operatorname{Ai}\left(s_{0}\right) \operatorname{Bi}^{\prime}\left(s_{1}\right)-\operatorname{Bi}\left(s_{0}\right) \operatorname{Ai}^{\prime}\left(s_{1}\right)\right]^{n+1}} \\
& -n s_{1} \int_{s_{0}}^{s_{1}} \frac{\operatorname{Bi}\left(s_{1}\right) \operatorname{Ai}(s)-\operatorname{Ai}\left(s_{1}\right) \operatorname{Bi}(s)}{\left[\operatorname{Bi}^{\prime}\left(s_{1}\right) \operatorname{Ai}(s)-\operatorname{Ai}^{\prime}\left(s_{1}\right) \operatorname{Bi}(s)\right]^{n+1}} \mathrm{~d} s
\end{aligned}
$$

for $n=2$ and $n=4$, obtained from (2.17) via Leibniz's rule together with the result

$$
\frac{\mathrm{d} s_{0}}{\mathrm{~d} s_{1}}=-\frac{s_{1}\left[\operatorname{Ai}^{\prime}\left(s_{0}\right) \operatorname{Bi}\left(s_{1}\right)-\operatorname{Bi}^{\prime}\left(s_{0}\right) \operatorname{Ai}\left(s_{1}\right)\right]}{s_{0}\left[\operatorname{Ai}\left(s_{0}\right) \operatorname{Bi}^{\prime}\left(s_{1}\right)-\operatorname{Bi}\left(s_{0}\right) \operatorname{Ai}^{\prime}\left(s_{1}\right)\right]},
$$

obtained by differentiation of $(2.16)_{1}$ with respect to $s_{1}$. Equations $(2.23)_{1}$ and $(2.29)$ are easily solved numerically to give $s_{0 \mathrm{c}} \simeq-0.883078$ and $s_{1 \mathrm{c}} \simeq 1.159888$, and then the critical value $b_{\mathrm{c}} \simeq 9.316786$ is recovered from $(2.23)_{2}$. The corresponding critical values of $\alpha$ and $\beta$ are $\alpha_{\mathrm{c}} \simeq 2.964360$ and $\beta_{\mathrm{c}} \simeq 0.353986 ;$ also $z\left(s_{0 \mathrm{c}}\right) \simeq 1.634701$ and $z\left(s_{1 \mathrm{c}}\right) \simeq 0.816886$, so that $h(0) \simeq 0.374217$ and $h(1) \simeq 1.498571$ for the critical solution.

\section{Properties of the solutions for $b \leq b_{\mathrm{c}}$}

The relation $z^{\prime \prime}(s)=s z(s)$ implies that $z^{\prime \prime}(s)$ is negative for $s \in\left[s_{0}, 0\right)$ and positive for $s \in\left(0, s_{1}\right]$. This, together with $(2.13)$, shows that $z^{\prime}(s)<0$ for $s \in\left(s_{0}, s_{1}\right)$. Hence $z(s)$ decreases monotonically from its maximum at $s=s_{0}$ to its minimum at $s=s_{1}$. Figure 4 shows a typical plot of $z$ as a function of $s \in\left[s_{0}, s_{1}\right]$, in the case $s_{1}=2.7$, for which $s_{0} \simeq-1.01722$. Since $x$ is an increasing function of $s$ by $(2.5)_{1}$, it follows from $(2.5)_{2}$ that $h(x)$ increases monotonically from its minimum at $x=0$ to its maximum at $x=1$. 


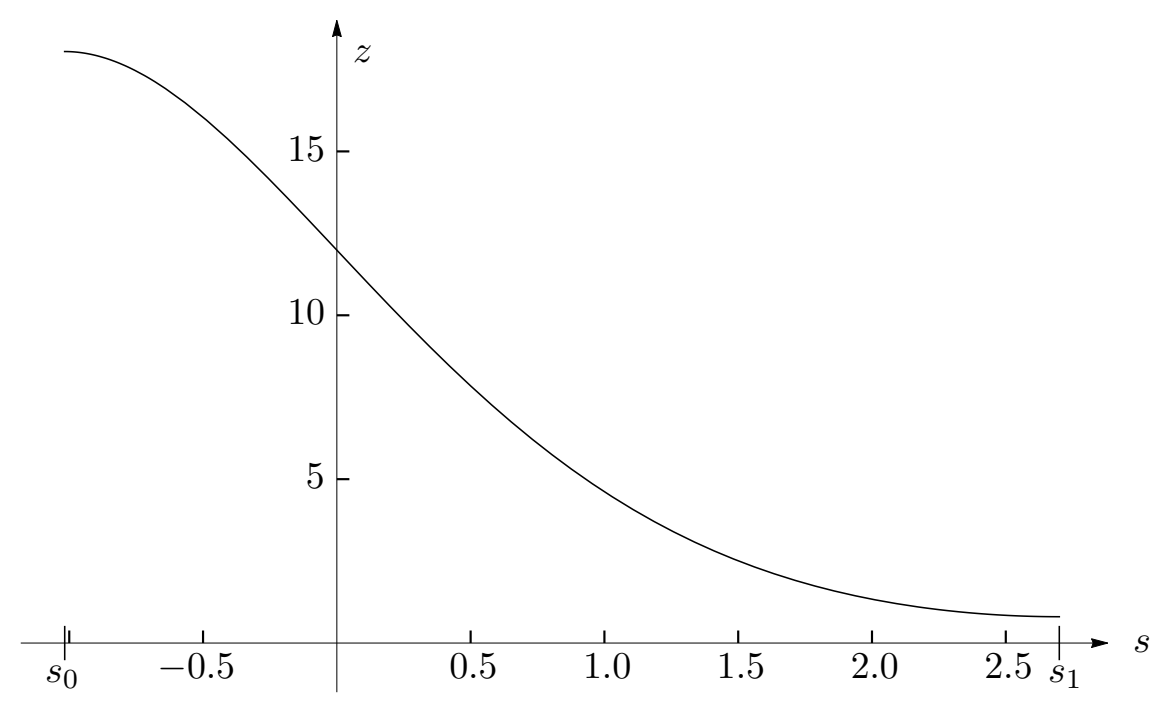

Figure 4: Typical plot of $z$ as a function of $s \in\left[s_{0}, s_{1}\right]$, in the case $s_{1}=2.7$, for which $s_{0} \simeq-1.01722$.

Relations $(2.10)_{2}$ and (2.13) imply that

$$
h^{\prime \prime}(0)=-2\left(\frac{b}{2}\right)^{2 / 3} s_{0}\left[z\left(s_{0}\right)\right]^{2}>0, \quad h^{\prime \prime}(1)=-2\left(\frac{b}{2}\right)^{2 / 3} s_{1}\left[z\left(s_{1}\right)\right]^{2}<0 .
$$

Moreover, $h^{\prime \prime \prime}(x)<0$ for $x \in(0,1)$ by the differential equation (1.7). Hence there exists exactly one $x_{\mathrm{i}} \in(0,1)$ such that $h^{\prime \prime}\left(x_{\mathrm{i}}\right)=0$, i.e. $h$ has exactly one inflection point. The corresponding parameter $s_{\mathrm{i}}$ satisfies $z^{\prime}\left(s_{\mathrm{i}}\right)^{2}=s_{\mathrm{i}} z\left(s_{\mathrm{i}}\right)^{2}$ by $(2.10)_{2}$, and therefore $s_{\mathrm{i}}>0$. Since $z>0$ and $z^{\prime}<0$ we have

$$
z^{\prime}\left(s_{\mathrm{i}}\right)=-\sqrt{s_{\mathrm{i}}} z\left(s_{\mathrm{i}}\right)
$$

This, together with $(2.10)_{1}$, gives the slope at the inflection point:

$$
h^{\prime}\left(x_{\mathrm{i}}\right)=2\left(\frac{b}{2}\right)^{1 / 3} \sqrt{s_{\mathrm{i}}} .
$$

Figure 5 shows plots of free surface profiles $h(x)$ obtained from $(2.24)-(2.25)$ in the cases (a) $b=0,1,2, \ldots, 8,9$ and $b=b_{\mathrm{c}}$, and corresponding to $s_{1} \in\left[0, s_{1 c}\right]$ (i.e. belonging to the first family of solutions) and (b) $b=1,2, \ldots, 8,9$ and $b=b_{\mathrm{c}}$, and corresponding to $s_{1} \in\left[s_{1 c}, \infty\right)$ (i.e. belonging to the second family of solutions). All the solutions for $b>0$ have the features discussed above: $h$ is a monotonically increasing function of $x$ with $h(0)>0$ and with a single inflection point in $(0,1)$; Karabut \& Pukhnachev [8] demonstrated the same properties for the first family of solutions.

From (1.10) and the monotonicity of $h$ it is clear that $T$ is a monotonically decreasing and convex function of $x$. Figures $6(\mathrm{a})$ and $6(\mathrm{~b})$ show the profiles of the reduced temperature $T(x) /|q|$ for the two families of solutions corresponding to the same values of $b$ as in Figures 5(a) and 5(b), respectively. 

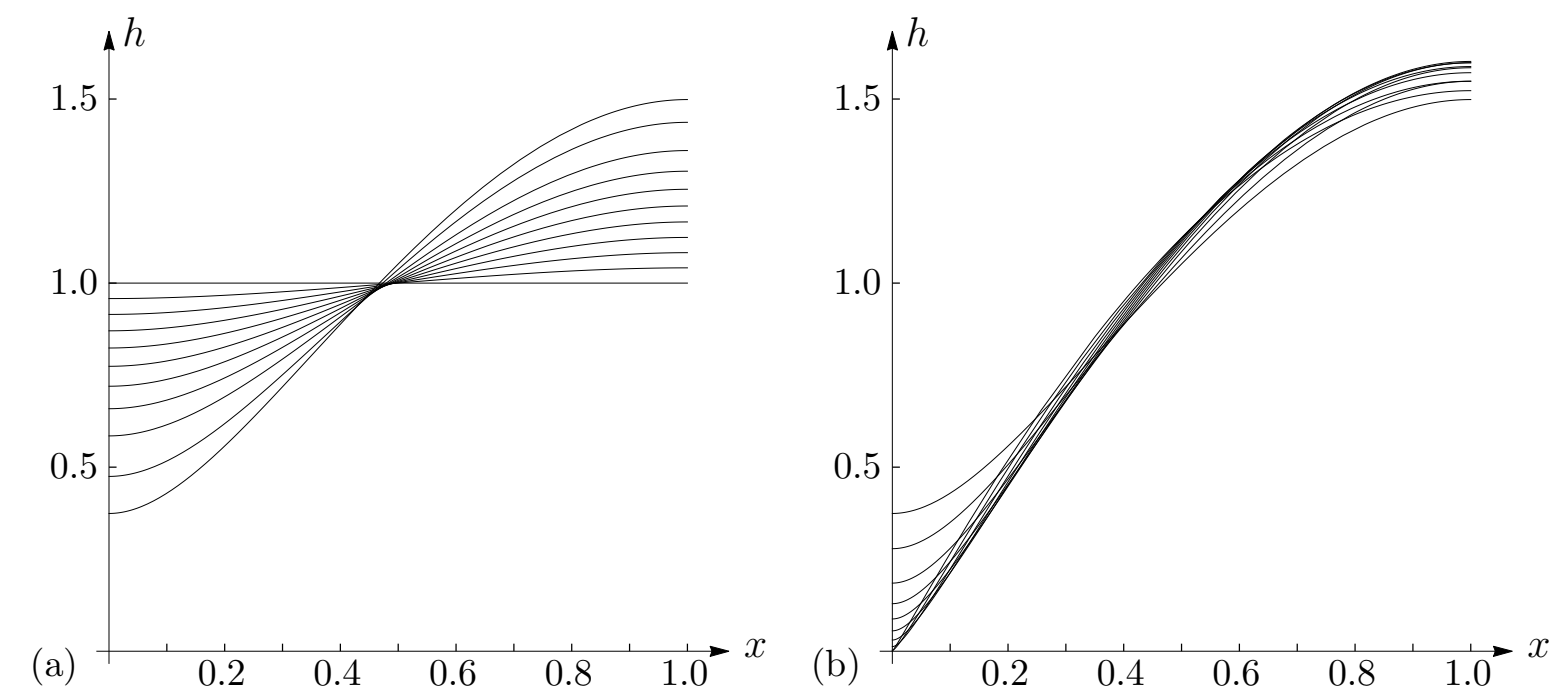

Figure 5: Plots of free surface profiles $h(x)$ in the cases (a) $b=0$ (for which $h \equiv 1$ ), 1, 2, $\ldots, 9$ and $b=b_{\mathrm{c}}$ belonging to the first family of solutions (cf. Figure 1 of [8]) and (b) $b=1$, $2, \ldots, 9$ and $b=b_{\mathrm{c}}$ belonging to the second family of solutions.

\section{Comparison with the results of Karabut \& Pukhnachev}

Karabut \& Pukhnachev [8, Section 3] obtained solutions of (1.7)-(1.9) numerically for $b<b^{*} \simeq 9.316$ which agree very well with our first family of solutions, cf. Figure $5(\mathrm{a})$ and [8, Figure 1]. Also our value of $b_{\mathrm{c}} \simeq 9.316786$ is in excellent agreement with their numerically calculated value of $b^{*}$, up to which they were able to solve the problem numerically.

However, at the end of their Section 2 Karabut \& Pukhnachev [8] claimed to have proved that the problem (1.7)-(1.9) has a solution for all positive $b$ (although it should be pointed out that they could not find solutions numerically for $\left.b>b^{*}\right)$. This conclusion clearly contradicts our Theorem 2.2. We believe the reason for this discrepancy is twofold. First, the signs in [8, equation (2.9)] seem to be wrong, which lead to different signs in the exponents in [8, equation (2.13)]. Secondly, the asymptotic expansion in [8, equation (2.20)] (specifically, the factor in front of the exponential function) seems to be wrong; see also the discussion at the end of this section. It is also worth pointing out that Karabut \& Pukhnachev [8] did not find our second family of solutions for $b<b_{\mathrm{c}}$, either numerically or analytically.

In their discussion of the properties of solutions of the system (1.7)-(1.11), Karabut \& Pukhnachev [8] first converted the problem to a pair of first order differential equations, given in their equations (2.6) and $(2.7) ;^{3}$ in fact, these differential equations may also be solved in closed (parametric) form. Specifically, for $c>0$ and $a \in \mathbb{R}, a \neq 0$, the initial value

\footnotetext{
${ }^{3}$ Equations (2.6) and (2.7) of Karabut \& Pukhnachev [8] correspond to the values $a=-1$ and $a=1$, respectively, in (4.1); however, we believe that these values are erroneous, and should be $a=-2$ and $a=2$, respectively.
} 

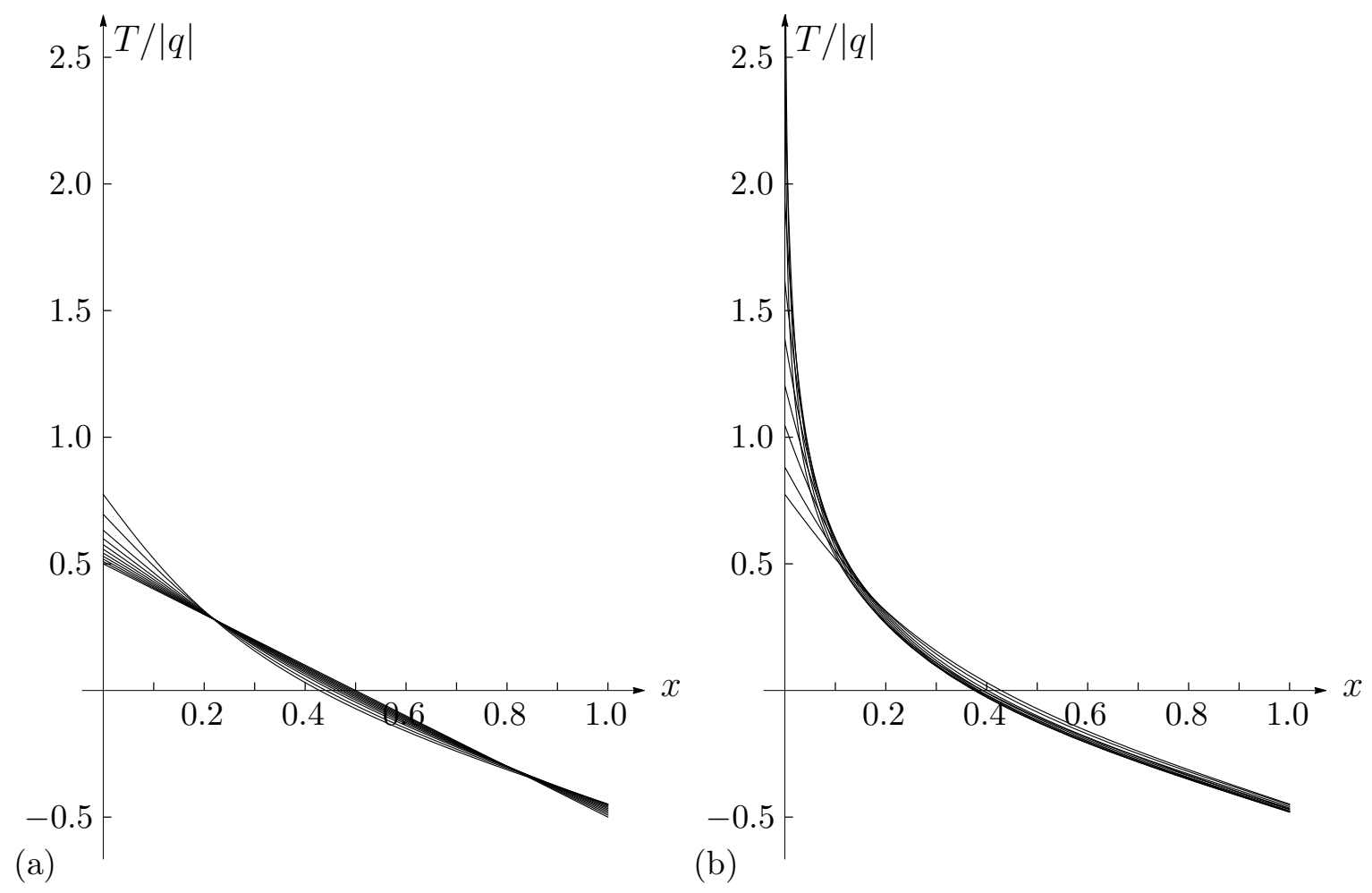

Figure 6: Plots of profiles of the reduced temperature $T(x) /|q|$ in the cases (a) $b=0$ (for which $\left.T(x) /|q|=\frac{1}{2}-x\right), 1,2, \ldots, 9$ and $b=b_{\mathrm{c}}$ belonging to the first family of solutions and (b) $b=1,2, \ldots, 9$ and $b=b_{\mathrm{c}}$ belonging to the second family of solutions.

problem

$$
\begin{aligned}
& \frac{\mathrm{d} w}{\mathrm{~d} \zeta}=-2+a \zeta \sqrt{w}, \quad \zeta \in(0, c), \\
& w(c)=0
\end{aligned}
$$

has a solution $w=w(\zeta)$ given in parametric form by

$$
\zeta=-\left(\frac{4}{|a|}\right)^{2 / 3} \frac{z^{\prime}(s)}{z(s)}, \quad w=\left(\frac{4}{|a|}\right)^{2 / 3}\left[\left(\frac{z^{\prime}(s)}{z(s)}\right)^{2}-s\right]^{2},
$$

where

$$
s \in\left[s_{0}, s_{\mathrm{i}}\right] \quad \text { if } a>0, \quad s \in\left[s_{\mathrm{i}}, s_{1}\right] \quad \text { if } a<0,
$$

$z$ is the function defined in $(2.25), s_{\mathrm{i}}$ is the unique positive solution of (3.2) and $s_{1}$ is such that

$$
c=\left(\frac{4}{|a|}\right)^{2 / 3} \sqrt{s_{\mathrm{i}}}
$$

It is always possible to find an $s_{1}$ such that (4.4) holds since one may show that $s_{\mathrm{i}} \rightarrow 0$ as $s_{1} \rightarrow 0^{+}$and that $s_{\mathrm{i}} \rightarrow \infty$ as $s_{1} \rightarrow \infty$.

Moreover, with the help of the solution (4.2) one may simplify the integrals that appear in [8, equation (2.13)]. Specifically, let $a_{0}>0, c>0, \eta \in \mathbb{R}$, and let $w_{1}$ and $w_{2}$ be the solutions (4.2) of (4.1) with $a=-a_{0}$ and $a=a_{0}$, respectively; then a straightforward calculation 
shows that

$$
\begin{aligned}
I_{\eta}(c) & :=\int_{0}^{c} \sum_{k=1}^{2} \exp \left[\eta(-1)^{k} \int_{\xi}^{c} \frac{\zeta}{\sqrt{w_{k}(\zeta)}} \mathrm{d} \zeta\right] \frac{1}{\sqrt{w_{k}(\xi)}} \mathrm{d} \xi \\
& =\left(\frac{4}{a_{0}}\right)^{1 / 3}\left[z\left(s_{\mathrm{i}}\right)\right]^{-4 \eta / a_{0}} \int_{s_{0}}^{s_{1}}[z(s)]^{4 \eta / a_{0}} \mathrm{~d} s \\
& \asymp\left[z\left(s_{\mathrm{i}}\right)\right]^{-4 \eta / a_{0}} s_{1}^{\eta / a_{0}} \exp \left(\frac{8 \eta}{3 a_{0}} s_{1}^{3 / 2}\right), \quad s_{1} \rightarrow \infty,
\end{aligned}
$$

where the notation $f(x) \asymp g(x)$ means that $f(x) / g(x)$ is both bounded and bounded away from 0 . Integrals of the above type arose in [8] in a discussion of existence of solutions of (1.7)-(1.9); specifically, it was claimed that the function $F(c):=\left[I_{1}(c)\right]^{2} / I_{2}(c)$ satisfies $F(c) \sim(2 \pi c)^{1 / 2} \rightarrow \infty$ as $c \rightarrow \infty$. However, from (4.5) we may now assert that $F(c) \asymp 1$ as $s_{1} \rightarrow \infty$ (that is, as $c \rightarrow \infty$ ), and so the claim in [8] that the equation $F(c)=b^{1 / 3}$ has a solution $c$ for any $b>0$ is unfounded.

\section{The limit $b \rightarrow 0^{+}$}

The simple solution (2.1) in the case $b=0$, which is included in Figures 5(a) and 6(a), is the limit of the first family of solutions of (1.7)-(1.11) when $b \rightarrow 0^{+}$, since from (2.26) we have $b \sim 16 s_{1}^{3} \rightarrow 0^{+}$in the limit $s_{1} \rightarrow 0^{+}$and hence $h(x) \rightarrow 1$ and $T(x) \rightarrow|q|\left(\frac{1}{2}-x\right)$ for every $x \in[0,1]$. A solution for small non-zero $b$ may be obtained as a regular expansion in $b$ about this solution (see Pukhnachev [14, equation (6.4)] and Karabut \& Pukhnachev [8, equations (1.11), (3.1)]), and is not repeated here, for brevity.

The limit as $b \rightarrow 0^{+}$of the second family of solutions of (1.7)-(1.11), which corresponds to $s_{1} \rightarrow \infty$, has a more complicated structure. From (2.21), (2.27) and (A.1) we obtain

$$
s_{0} \sim \hat{s}_{0}+\frac{\pi\left[\operatorname{Bi}^{\prime}\left(\hat{s}_{0}\right)\right]^{2}}{2\left|\hat{s}_{0}\right|} \exp \left(-\frac{4}{3} s_{1}^{3 / 2}\right), \quad s_{1} \rightarrow \infty,
$$

where $\hat{s}_{0}$ is again the largest (negative) zero of $\operatorname{Ai}^{\prime}(s)$. In particular, $s_{0} \rightarrow \hat{s}_{0}^{+}$as $s_{1} \rightarrow \infty$. Now Lemma A.1 and equation (A.1) yield

$$
\begin{aligned}
z\left(s_{0}\right) & =\frac{J_{4}^{1 / 2}}{J_{2}^{1 / 2}}\left(\operatorname{Bi}^{\prime}\left(s_{1}\right) \operatorname{Ai}\left(s_{0}\right)-\operatorname{Ai}^{\prime}\left(s_{1}\right) \operatorname{Bi}\left(s_{0}\right)\right) \\
& \sim \frac{\sqrt{2 / 3} \pi^{2} s_{1}^{-1 / 4}}{\pi s_{1}^{-1 / 4}} \operatorname{Ai}\left(\hat{s}_{0}\right) \operatorname{Bi}^{\prime}\left(s_{1}\right) \\
& \sim \sqrt{\frac{2 \pi}{3}} \operatorname{Ai}\left(\hat{s}_{0}\right) s_{1}^{1 / 4} \exp \left(\frac{2}{3} s_{1}^{3 / 2}\right) .
\end{aligned}
$$

Hence

$$
h(0)=\frac{1}{\left[z\left(s_{0}\right)\right]^{2}} \sim \frac{3}{2 \pi\left[\operatorname{Ai}\left(\hat{s}_{0}\right)\right]^{2}} s_{1}^{-1 / 2} \exp \left(-\frac{4}{3} s_{1}^{3 / 2}\right), \quad s_{1} \rightarrow \infty
$$


with $b$ and $s_{1}$ related by (2.27); thus the thickness $2 h$ at the left endpoint $x=0$ decays super-exponentially with $s_{1}$. Also the curvature at this endpoint grows super-exponentially with $s_{1}$ since, by $(3.1)_{1}$,

$$
\begin{aligned}
h^{\prime \prime}(0) & =-2\left(\frac{b}{2}\right)^{2 / 3} s_{0}\left[z\left(s_{0}\right)\right]^{2} \\
& \sim 3 \pi\left|\hat{s}_{0}\right|\left[\operatorname{Ai}\left(\hat{s}_{0}\right)\right]^{2} s_{1}^{-1 / 2} \exp \left(\frac{4}{3} s_{1}^{3 / 2}\right), \quad s_{1} \rightarrow \infty .
\end{aligned}
$$

We show that, in the limit $b \rightarrow 0^{+}$, the second family of solutions converges to the function

$$
h_{0}(x)=\frac{3}{2} x(2-x), \quad x \in[0,1] .
$$

To this end we use the substitution

$$
s=s_{1}-s_{1}^{-1 / 2} t, \quad 0 \leq t \leq s_{1}^{3 / 2}-s_{0} s_{1}^{1 / 2},
$$

as in the proof of Lemma A.1. For fixed $t$ we have

$$
z\left(s_{1}-s_{1}^{-1 / 2} t\right) \rightarrow \sqrt{\frac{2}{3}} \cosh t, \quad s_{1} \rightarrow \infty
$$

by (A.2) and (A.18), which implies that

$$
h \rightarrow \frac{3}{2 \cosh ^{2} t}, \quad s_{1} \rightarrow \infty .
$$

With (5.2), (5.7) and (A.17) we obtain

$$
\begin{aligned}
x & =\pi\left(\frac{2}{b}\right)^{1 / 3} \frac{\operatorname{Ai}\left(s_{0}\right) \operatorname{Bi}\left(s_{1}-s_{1}^{-1 / 2} t\right)-\operatorname{Ai}\left(s_{1}-s_{1}^{-1 / 2} t\right) \operatorname{Bi}\left(s_{0}\right)}{z\left(s_{0}\right) z\left(s_{1}-s_{1}^{-1 / 2} t\right)} \\
& \sim \pi \frac{2}{3} s_{1}^{1 / 2} \frac{\operatorname{Ai}\left(\hat{s}_{0}\right) \pi^{-1 / 2} s_{1}^{-1 / 4} \exp \left[(2 / 3)\left(s_{1}-s_{1}^{-1 / 2} t\right)^{3 / 2}\right]}{\sqrt{2 \pi / 3} \operatorname{Ai}\left(\hat{s}_{0}\right) s_{1}^{1 / 4} \exp \left[(2 / 3) s_{1}^{3 / 2}\right] \sqrt{2 / 3} \cosh t} \\
& \sim \frac{1}{\cosh t} \exp \left[\frac{2}{3}\left(\left(s_{1}-s_{1}^{-1 / 2} t\right)^{3 / 2}-s_{1}^{3 / 2}\right)\right] \rightarrow \frac{\mathrm{e}^{t}}{\cosh t}, \quad s_{1} \rightarrow \infty .
\end{aligned}
$$

Since

$$
\frac{3 \mathrm{e}^{t}}{2 \cosh t}\left(2-\frac{\mathrm{e}^{t}}{\cosh t}\right)=\frac{3}{2 \cosh ^{2} t},
$$

it follows from (5.8) and (5.9) that the second family of solutions of (1.7)-(1.9) converges to $h_{0}$ given in (5.5) as $b \rightarrow 0$, i.e.

$$
h(x) \rightarrow \frac{3}{2} x(2-x), \quad x \in[0,1]
$$

as $s_{1} \rightarrow \infty$. The function $h_{0}$ satisfies the differential equation (1.7), the boundary condition $(1.8)_{2}$ at $x=1$ and the volume condition (1.9). However, this "outer" solution does not satisfy the boundary condition $(1.8)_{1}$ at $x=0$, and there is an "inner" solution in a boundary layer near $x=0$ that accommodates this boundary condition; this is in accord with the divergence of $h^{\prime \prime}(0)$ in this limit. 


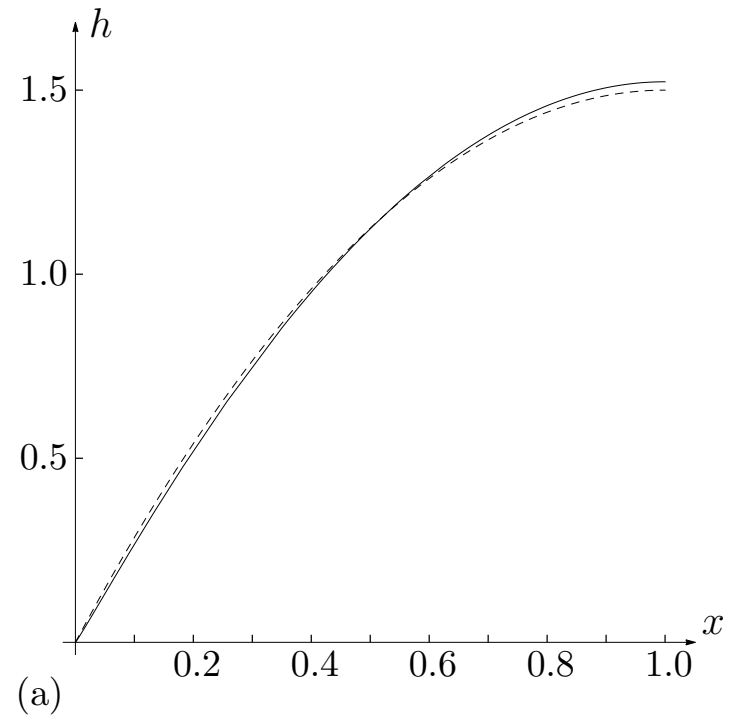

(a)

(b)

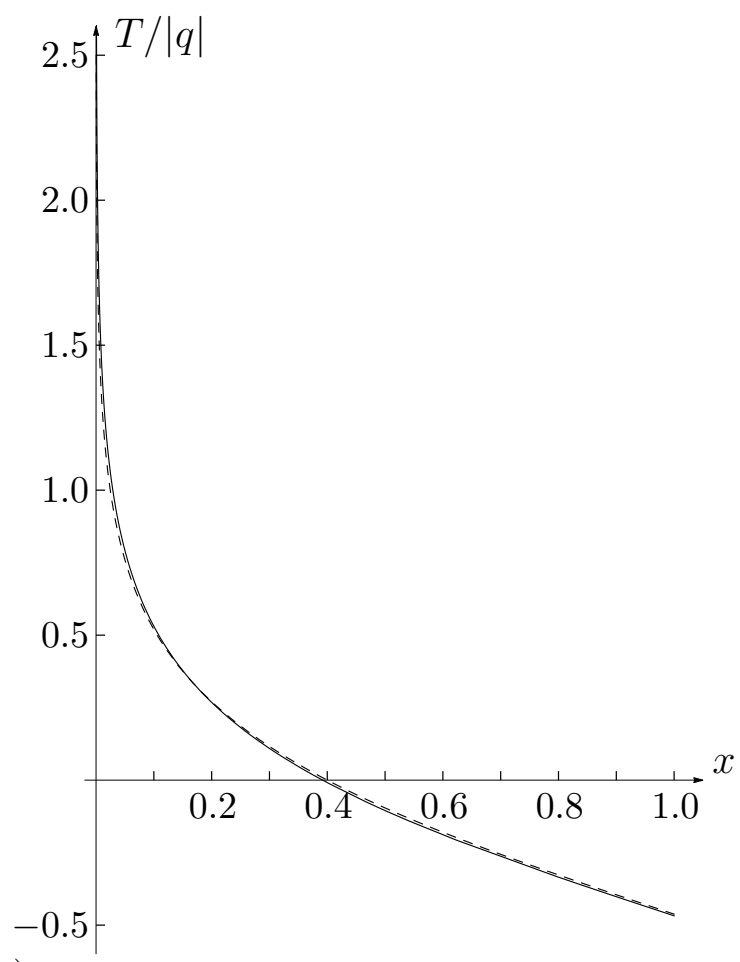

Figure 7: Plots of profiles of (a) the free surface $h(x)$ and (b) the reduced temperature $T(x) /|q|$ of the second family of solutions in the case $b=1$ (full curves) and the leading order outer asymptotic solutions in the limit $b \rightarrow 0$ given by (5.11) and (5.12) (dashed curves).

In a similar way to the above one can show that the temperature converges for $x \in(0,1]$ and diverges for $x=0$ :

$$
\begin{aligned}
& T(x) \rightarrow \frac{|q|}{3} \log \left(\frac{2-x}{4 x}\right), \quad x \in(0,1], \\
& T(0) \sim \frac{2|q|}{3} s_{1}^{3 / 2} \rightarrow \infty, \quad s_{1} \rightarrow \infty .
\end{aligned}
$$

Figure 7 shows comparisons between the asymptotic outer solutions for $h(x)$ and $T(x) /|q|$ given in (5.11) and (5.12) and the exact solutions (2.24) and (2.9) in the case $b=1$ belonging to the second family, i.e. with $s_{1} \simeq 3.9532>s_{1 \mathrm{c}}$. The agreement is very good, especially since the value $b=1$ is not particularly small, given that $b$ takes values only in the interval $0 \leq b \leq b_{\mathrm{c}} \simeq 9.316786$. Physically the solution (5.5) may be interpreted as representing a situation in which the effective Marangoni number $\gamma$ is small (so that the surface tension is essentially constant) and in which the free surface has constant curvature except near $x=0$ where it distorts strongly to satisfy the contact-angle condition $h^{\prime}(0)=0$. Equation $(1.6)_{1}$ shows that for the curvature to be non-constant near $x=0$ the temperature gradient must be large there (and so thermocapillarity is significant), and $(1.6)_{2}$ shows that this is achieved with small $h$. 


\section{Conclusions}

We have obtained a closed-form (parametric) solution of the steady two-dimensional thinfilm version of a problem concerning a weightless non-isothermal free film of incompressible viscous fluid subject to thermocapillarity, proposed and analysed by Pukhnachev and coworkers, and defined here in equations (1.7)-(1.11). Specifically, we extended and corrected the paper by Karabut \& Pukhnachev [8] in which the problem is solved numerically, and in which it is claimed that there exists a unique solution for any value of $b$. We showed that, on the contrary, solutions exist only when $b \leq b_{\mathrm{c}} \simeq 9.316786$, and that there are then two solutions, one of which recovers that obtained numerically by Karabut \& Pukhnachev [8], the other being new.

The questions of why, physically, the value $b=b_{\mathrm{c}}$ is critical, and of what happens when $b>$ $b_{\mathrm{c}}$, remain open. It is conceivable that an unsteady evolution develops for $b>b_{\mathrm{c}}$, maintained energetically by the heat input/output at the planes $x=0$ and $x=1$; the equations derived by Pukhnachev \& Dubinkina [15] for the unsteady situation would presumably be the starting point for an analysis of such evolutions. It is also conceivable that the steady solutions in the second family derived above are unstable; however, this is untested as yet.

We have followed Pukhnachev and co-workers in taking the film to be symmetric with respect to the plane $z=0$; it would be of interest to determine whether steady nonsymmetric solutions are also possible.

One advantage of having a closed-form solution is that we have been able to use it to prove that the curve in Figure 3 satisfies (a) $b \rightarrow 0$ as $s_{1} \rightarrow 0$, (b) $b$ has a global maximum $b=b_{\mathrm{c}}$ at $s=s_{1 \mathrm{c}}$, and (c) $b \rightarrow 0$ as $s_{1} \rightarrow \infty$, and therefore that there are no "higher" branches of solutions beyond those shown in Figure 3. However, we have not been able to prove that the curve behaves monotonically on either side of its global maximum; if it does not then for some given values of $b\left(<b_{\mathrm{c}}\right)$ there will be more than two associated values of $s_{1}$, and hence there will be more than two solutions of the original problem.

The question of the possibility of the film "pinching off" when the two free surfaces come into contact is also of interest. Pukhnachev [13] showed that the solutions in the first family never approach pinch-off; this is consistent with Figure 5(a), which shows that even for the maximum value $b=b_{\mathrm{c}}$ the film is of finite thickness everywhere. We have shown that the solutions in the second family approach pinch-off only in the limit $b \rightarrow 0$, and then only at $x=0$.

\section{A Appendix}

In this appendix we establish the asymptotic behaviour of the integrals $J_{2}$ and $J_{4}$ defined in (2.17) in the limit $s_{1} \rightarrow \infty$. To this end let us first recall asymptotic relations for $\mathrm{Ai}, \mathrm{Bi}$ 
and their derivatives (see, e.g. [12, 9.7.5-9.7.8]):

$$
\begin{array}{ll}
\operatorname{Ai}(s) \sim \frac{1}{2 \sqrt{\pi}} s^{-1 / 4} \exp \left(-\frac{2}{3} s^{3 / 2}\right), & \operatorname{Ai}^{\prime}(s) \sim-\frac{1}{2 \sqrt{\pi}} s^{1 / 4} \exp \left(-\frac{2}{3} s^{3 / 2}\right), \\
\operatorname{Bi}(s) \sim \frac{1}{\sqrt{\pi}} s^{-1 / 4} \exp \left(\frac{2}{3} s^{3 / 2}\right), & \operatorname{Bi}^{\prime}(s) \sim \frac{1}{\sqrt{\pi}} s^{1 / 4} \exp \left(\frac{2}{3} s^{3 / 2}\right)
\end{array}
$$

as $s \rightarrow \infty$. The following lemma gives the desired asymptotic behaviour of $J_{2}$ and $J_{4}$.

Lemma A.1. For given $s_{1}>0$ let $s_{0}$ be the unique solution of $(2.23)_{1}$ in $\left(\hat{s}_{0}, 0\right)$. Then

$$
J_{2}\left(s_{0}, s_{1}\right) \sim \pi^{2} s_{1}^{-1 / 2}, \quad J_{4}\left(s_{0}, s_{1}\right) \sim \frac{2 \pi^{4}}{3} s_{1}^{-1 / 2}, \quad s_{1} \rightarrow \infty .
$$

Proof. From (2.20) and (2.6) it may be shown that

$$
J_{2}\left(s_{0}, s_{1}\right)=\pi^{2} \frac{\operatorname{Bi}\left(s_{1}\right)}{\operatorname{Bi}^{\prime}\left(s_{1}\right)}-\frac{\pi \operatorname{Bi}\left(s_{0}\right)}{\operatorname{Bi}^{\prime}\left(s_{1}\right)\left[\operatorname{Bi}^{\prime}\left(s_{1}\right) \operatorname{Ai}\left(s_{0}\right)-\operatorname{Ai}^{\prime}\left(s_{1}\right) \operatorname{Bi}\left(s_{0}\right)\right]} .
$$

The result for $J_{2}$ in (A.2) follows from (A.3) and (A.1), the second term in (A.3) decaying exponentially.

For $J_{4}$ first note that

$$
\operatorname{Ai}(s), \operatorname{Bi}(s), \operatorname{Bi}^{\prime}(s)>0 \quad \text { and } \quad \operatorname{Ai}^{\prime}(s)<0, \quad s \in\left(\hat{s}_{0}, \infty\right)
$$

Using (A.4) we may estimate the integral over the negative part of the interval as follows:

$$
\begin{aligned}
& \int_{s_{0}}^{0} \frac{1}{\left[\operatorname{Bi}^{\prime}\left(s_{1}\right) \operatorname{Ai}(s)-\operatorname{Ai}^{\prime}\left(s_{1}\right) \operatorname{Bi}(s)\right]^{4}} \mathrm{~d} s \leq \int_{\hat{s}_{0}}^{0} \frac{1}{\left[\operatorname{Bi}^{\prime}\left(s_{1}\right) \operatorname{Ai}(s)\right]^{4}} \mathrm{~d} s \\
& =\frac{1}{\left[\operatorname{Bi}^{\prime}\left(s_{1}\right)\right]^{4}} \int_{\hat{s}_{0}}^{0} \frac{1}{[\operatorname{Ai}(s)]^{4}} \mathrm{~d} s \leq \frac{C}{s_{1}} \exp \left(-\frac{8}{3} s_{1}^{3 / 2}\right),
\end{aligned}
$$

which is exponentially small.

Now consider the second part of the integral. Let us define

$$
\widetilde{J}_{4}\left(s_{1}\right):=\int_{0}^{s_{1}} \frac{1}{\left[\operatorname{Bi}^{\prime}\left(s_{1}\right) \operatorname{Ai}(s)-\operatorname{Ai}^{\prime}\left(s_{1}\right) \operatorname{Bi}(s)\right]^{4}} \mathrm{~d} s
$$

for $s_{1}>0$. In this integral we make the substitution $s=s_{1}-s_{1}^{-1 / 2} t$, which yields

$$
\widetilde{J}_{4}\left(s_{1}\right)=s_{1}^{-1 / 2} \int_{0}^{s_{1}^{3 / 2}} \frac{\mathrm{d} t}{\left[\operatorname{Bi}^{\prime}\left(s_{1}\right) \operatorname{Ai}\left(s_{1}-s_{1}^{-1 / 2} t\right)-\operatorname{Ai}^{\prime}\left(s_{1}\right) \operatorname{Bi}\left(s_{1}-s_{1}^{-1 / 2} t\right)\right]^{4}} .
$$

We show that the integrand is uniformly bounded by an integrable function so that we may apply the Dominated Convergence Theorem. It follows from the asymptotic formulae (A.1) that there exist $m, M>0$ such that

$$
\begin{gathered}
m(1+s)^{1 / 4} \exp \left(\frac{2}{3} s^{3 / 2}\right) \leq \operatorname{Bi}^{\prime}(s) \leq M(1+s)^{1 / 4} \exp \left(\frac{2}{3} s^{3 / 2}\right), \\
m(1+s)^{-1 / 4} \exp \left(-\frac{2}{3} s^{3 / 2}\right) \leq \mathrm{Ai}(s) \leq M(1+s)^{-1 / 4} \exp \left(-\frac{2}{3} s^{3 / 2}\right)
\end{gathered}
$$


for all $s \in[0, \infty)$. Hence for $0 \leq t \leq s_{1}^{3 / 2}$ we have

$$
\begin{aligned}
& \operatorname{Bi}^{\prime}\left(s_{1}\right) \operatorname{Ai}\left(s_{1}-s_{1}^{-1 / 2} t\right)-\operatorname{Ai}^{\prime}\left(s_{1}\right) \operatorname{Bi}\left(s_{1}-s_{1}^{-1 / 2} t\right) \\
& \geq \operatorname{Bi}^{\prime}\left(s_{1}\right) \operatorname{Ai}\left(s_{1}-s_{1}^{-1 / 2} t\right) \\
& \geq m^{2}\left(1+s_{1}\right)^{1 / 4} \exp \left(\frac{2}{3} s_{1}^{3 / 2}\right)\left(1+s_{1}-s_{1}^{-1 / 2} t\right)^{-1 / 4} \exp \left(-\frac{2}{3}\left(s_{1}-s_{1}^{-1 / 2} t\right)^{3 / 2}\right) \\
& =m^{2}\left(1-\frac{t}{\left(1+s_{1}\right) s_{1}^{1 / 2}}\right)^{-1 / 4} \exp \left(\frac{2}{3}\left[s_{1}^{3 / 2}-\left(s_{1}-s_{1}^{-1 / 2} t\right)^{3 / 2}\right]\right) \\
& \geq m^{2} \exp \left(f\left(s_{1}, t\right)\right),
\end{aligned}
$$

where use has been made of (A.4), and we have introduced the function

$$
f\left(s_{1}, t\right):=\frac{2}{3}\left[s_{1}^{3 / 2}-\left(s_{1}-s_{1}^{-1 / 2} t\right)^{3 / 2}\right], \quad s_{1}, t \in[0, \infty), s_{1} \geq t^{2 / 3} .
$$

The derivative of $f\left(s_{1}, t\right)$ with respect to $s_{1}$ is given by

$$
\begin{aligned}
\frac{\partial f\left(s_{1}, t\right)}{\partial s_{1}} & =s_{1}^{1 / 2}-\left(s_{1}-s_{1}^{-1 / 2} t\right)^{1 / 2}\left(1+\frac{1}{2} s_{1}^{-3 / 2} t\right) \\
& =\frac{t^{2}\left(3+s_{1}^{-3 / 2} t\right)}{4 s_{1}^{2}\left[s_{1}^{1 / 2}+\left(s_{1}-s_{1}^{-1 / 2} t\right)^{1 / 2}\left(1+\frac{1}{2} s_{1}^{-3 / 2} t\right)\right]}
\end{aligned}
$$

showing that $\partial f\left(s_{1}, t\right) / \partial s_{1} \geq 0$, which in turn implies that

$$
f\left(s_{1}, t\right) \geq f\left(t^{2 / 3}, t\right)=\frac{2}{3}\left[t-\left(t^{2 / 3}-t^{-1 / 3} t\right)^{3 / 2}\right]=\frac{2}{3} t \quad \text { for } \quad s_{1} \geq t^{2 / 3} .
$$

It therefore follows from (A.10) and (A.13) that

$$
\operatorname{Bi}^{\prime}\left(s_{1}\right) \operatorname{Ai}\left(s_{1}-s_{1}^{-1 / 2} t\right)-\operatorname{Ai}^{\prime}\left(s_{1}\right) \operatorname{Bi}\left(s_{1}-s_{1}^{-1 / 2} t\right) \geq m^{2} \exp \left(f\left(s_{1}, t\right)\right) \geq m^{2} \exp \left(\frac{2}{3} t\right) .
$$

Hence the integrand in (A.7) is bounded from above by

$$
\frac{1}{m^{8}} \exp \left(-\frac{8}{3} t\right)
$$

which is an integrable function. Let us consider the pointwise limit of the integrand as $s_{1} \rightarrow \infty$, i.e. for fixed $t$. From the asymptotic expansions in (A.1) we obtain

$$
\begin{aligned}
& \operatorname{Bi}^{\prime}\left(s_{1}\right) \operatorname{Ai}\left(s_{1}-s_{1}^{-1 / 2} t\right)-\operatorname{Ai}^{\prime}\left(s_{1}\right) \operatorname{Bi}\left(s_{1}-s_{1}^{-1 / 2} t\right) \\
& \sim \frac{1}{2 \pi} s_{1}^{1 / 4} \exp \left(\frac{2}{3} s_{1}^{3 / 2}\right)\left(s_{1}-s_{1}^{-1 / 2} t\right)^{-1 / 4} \exp \left(-\frac{2}{3}\left(s_{1}-s_{1}^{-1 / 2} t\right)^{3 / 2}\right) \\
& \quad+\frac{1}{2 \pi} s_{1}^{1 / 4} \exp \left(-\frac{2}{3} s_{1}^{3 / 2}\right)\left(s_{1}-s_{1}^{-1 / 2} t\right)^{-1 / 4} \exp \left(\frac{2}{3}\left(s_{1}-s_{1}^{-1 / 2} t\right)^{3 / 2}\right) \\
& =\frac{1}{2 \pi}\left(1-s_{1}^{-3 / 2} t\right)^{-1 / 4}\left[\exp \left(f\left(s_{1}, t\right)\right)+\exp \left(-f\left(s_{1}, t\right)\right)\right] \\
& \sim \frac{1}{\pi} \cosh \left(f\left(s_{1}, t\right)\right) .
\end{aligned}
$$


The function $f\left(s_{1}, t\right)$ has the asymptotic behaviour

$$
\begin{aligned}
f\left(s_{1}, t\right) & =\frac{2}{3} s_{1}^{3 / 2}\left[1-\left(1-s_{1}^{-3 / 2} t\right)^{3 / 2}\right] \\
& =t+\mathrm{O}\left(s_{1}^{-3 / 2}\right) \rightarrow t, \quad s_{1} \rightarrow \infty
\end{aligned}
$$

and hence

$$
\operatorname{Bi}^{\prime}\left(s_{1}\right) \operatorname{Ai}\left(s_{1}-s_{1}^{-1 / 2} t\right)-\operatorname{Ai}^{\prime}\left(s_{1}\right) \operatorname{Bi}\left(s_{1}-s_{1}^{-1 / 2} t\right) \rightarrow \frac{1}{\pi} \cosh t, \quad s_{1} \rightarrow \infty .
$$

By the Dominated Convergence Theorem we therefore obtain

$$
\lim _{s_{1} \rightarrow \infty} \int_{0}^{s_{1}^{3 / 2}} \frac{\mathrm{d} t}{\left[\operatorname{Bi}^{\prime}\left(s_{1}\right) \operatorname{Ai}\left(s_{1}-s_{1}^{-1 / 2} t\right)-\operatorname{Ai}^{\prime}\left(s_{1}\right) \operatorname{Bi}\left(s_{1}-s_{1}^{-1 / 2} t\right)\right]^{4}}=\pi^{4} \int_{0}^{\infty} \frac{\mathrm{d} t}{\cosh ^{4} t} .
$$

With the substitution $x=\mathrm{e}^{2 t}$ we have

$$
\int_{0}^{\infty} \frac{\mathrm{d} t}{\cosh ^{4} t}=\int_{0}^{\infty} \frac{16 \mathrm{e}^{4 t}}{\left(1+\mathrm{e}^{2 t}\right)^{4}} \mathrm{~d} t=\int_{1}^{\infty} \frac{8 x}{(1+x)^{4}} \mathrm{~d} x=\frac{2}{3}
$$

and hence

$$
\lim _{s_{1} \rightarrow \infty} \int_{0}^{s_{1}^{3 / 2}} \frac{\mathrm{d} t}{\left[\operatorname{Bi}^{\prime}\left(s_{1}\right) \operatorname{Ai}\left(s_{1}-s_{1}^{-1 / 2} t\right)-\operatorname{Ai}^{\prime}\left(s_{1}\right) \operatorname{Bi}\left(s_{1}-s_{1}^{-1 / 2} t\right)\right]^{4}}=\frac{2 \pi^{4}}{3},
$$

which, together with (A.5) and (A.7), implies that

$$
J_{4}\left(s_{0}, s_{1}\right) \sim \widetilde{J}_{4}\left(s_{1}\right) \sim \frac{2 \pi^{4}}{3} s_{1}^{-1 / 2}, \quad s_{1} \rightarrow \infty .
$$

\section{Acknowledgements}

This work was completed while S. K. W. was a Leverhulme Trust Research Fellow (20132016) supported by award RF-2013-355, "Small Particles, Big Problems: Understanding the Complex Behaviour of Nanofluids".

\section{References}

[1] Abramowitz, M. \& Stegun, I. A. (1964) Handbook of Mathematical Functions. Dover.

[2] Bonn, D., Eggers, J., Indekeu, J., Meunier, J. \& Rolley, E. (2009) Wetting and spreading. Rev. Mod. Phys. 81, 739-805.

[3] Chan, T. S., Gueudré, T. \& Snoeijer, J. H. (2011) Maximum speed of dewetting on a fiber. Phys. Fluids 23, 112103, 9pp. 
[4] Duffy, B. R. \& Wilson, S. K. (1997) A third-order differential equation arising in thin-film theory and relevant to Tanner's law. Appl. Math. Lett. 10, 63-68.

[5] Eggers, J. (2004) Hydrodynamic theory of forced dewetting. Phys. Rev. Lett. 93, 094502, 4pp.

[6] Eggers, J. (2005) Existence of receding and advancing contact lines. Phys. Fluids 17, 082106, 10pp.

[7] Janeček, V., Andreotti, B., Pražák, D., Bárta, T. \& Nikolayev, V. S. (2013) Moving contact line of a volatile fluid. Phys. Rev. E 88, 060404, 5pp.

[8] Karabut, E. A. \& Pukhnachev, V. V. (2008) Steady-state conditions of a nonisothermal film with a heat-insulated free boundary. J. App. Mech. Tech. Phys. 49, $568-579$.

[9] Karpitschka, S. \& Riegler, H. (2012) Noncoalescence of sessile drops from different but miscible liquids: hydrodynamic analysis of the twin drop contour as a selfstabilizing traveling wave. Phys. Rev. Lett. 109, 066103, 5pp.

[10] Limat, L. \& Stone, H. A. (2004) Three-dimensional lubrication model of a contact line corner singularity. Europhys. Lett. 65, 365-371.

[11] Neogi, P. (2010) Bead formation near the contact line in forced spreading. Chem. Eng. Sci. 65, 4572-4578.

[12] NIST Handbook of Mathematical Functions. Edited by F. W. J. Olver, D. W. Lozier, R. F. Boisvert and C.W. Clark. U.S. Department of Commerce, National Institute of Standards and Technology, Washington, DC; Cambridge University Press, Cambridge (2010). Online version: http://dlmf.nist.gov

[13] Pukhnachov, V. V. (2002) Model of a viscous layer deformation by thermocapillary forces. European J. Appl. Math. 13, 205-224.

[14] Pukhnachev, V. V. (2007) Equilibrium of a free nonisothermal liquid film. J. App. Mech. Tech. Phys. 48, 310-321.

[15] Pukhnachev, V. V. \& Dubinkina, S. B. (2006) A model of film deformation and rupture under the action of thermocapillary forces. Fluid Dynamics 41, 755-771.

[16] Snoeijer, J. H. \& Andreotti, B. (2013) Moving contact lines: scales, regimes, and dynamical transitions. Annu. Rev. Fluid Mech. 45, 269-292

[17] Tuck, E. O. \& Schwartz, L. W. (1990) A numerical and asymptotic study of some third-order ordinary differential equations relevant to draining and coating flows. SIAM Review 32, 453-469.

[18] Voinov, O. V. (1977) Inclination angles of the boundary in moving liquid layers. $Z h$. Prikl. Mekh. Tekh. 2, 92-99. 